\title{
Baseline Oxidative Stress Is Associated with Memory Changes in Omega-3 Fatty Acid Treated Coronary Artery Disease Patients
}

\author{
Graham Mazereeuw, ${ }^{1}$ Nathan Herrmann, ${ }^{2}$ Ana C. Andreazza, ${ }^{3}$ Gustavo Scola, \\ David W. L. Ma, ${ }^{5}$ Paul I. Oh, ${ }^{6}$ and Krista L. Lanctôt ${ }^{7}$ \\ ${ }^{1}$ MD Program, University of Toronto, Toronto, ON, Canada \\ ${ }^{2}$ Hurvitz Brain Sciences Program, Sunnybrook Research Institute and Department of Psychiatry, University of Toronto, \\ Toronto, ON, Canada \\ ${ }^{3}$ Centre for Addiction and Mental Health and Departments of Psychiatry and Pharmacology/Toxicology, University of Toronto, \\ Toronto, ON, Canada \\ ${ }^{4}$ Centre for Addiction and Mental Health and Department of Psychiatry, University of Toronto, Toronto, ON, Canada \\ ${ }^{5}$ Department of Human Health and Nutritional Sciences, University of Guelph, Guelph, ON, Canada \\ ${ }^{6}$ University Health Network at Toronto Rehabilitation Institute, Toronto, ON, Canada \\ ${ }^{7}$ Hurvitz Brain Sciences Program, Sunnybrook Research Institute and Departments of Psychiatry and Pharmacology/Toxicology, \\ University of Toronto, Toronto, ON, Canada \\ Correspondence should be addressed to Krista L. Lanctôt; krista.lanctot@sunnybrook.ca
}

Received 17 July 2017; Revised 19 September 2017; Accepted 28 September 2017; Published 2 November 2017

Academic Editor: Janusz K. Rybakowski

Copyright (C) 2017 Graham Mazereeuw et al. This is an open access article distributed under the Creative Commons Attribution License, which permits unrestricted use, distribution, and reproduction in any medium, provided the original work is properly cited.

Objective. This study investigated whether pretreatment oxidative stress, measured by lipid hydroperoxides (LPH), 4-hydroxy-2nonenal (4-HNE), 8-isoprostane (8-ISO), and malondialdehyde (MDA), was associated with improvement in immediate recall among n-3 PUFA-treated coronary artery disease patients. Methods. This was a secondary analysis of the CAROTID trial (NCT00981383). Composite immediate recall, measured using the California Verbal Learning Test, Second Edition, and the Brief Visuospatial Memory Test-Revised, was assessed. LPH, 4-HNE, 8-ISO, MDA, and n-3 PUFA concentrations were analysed from fasting blood. Patients then received either n-3 PUFA treatment or placebo for 12 weeks, after which composite immediate recall was reassessed. Linear regression was used to investigate relationships between lipid peroxidation markers and changes in composite immediate recall in each treatment group. Results. Eighty-five patients (age $=61.1 \pm 8.5,77 \%$ male, mean years of education $=$ $15.3 \pm 3.4$ ) were included ( $n=46$ placebo, $n=39$ n-3 PUFA). After adjusting for multiple comparisons and potential confounders, greater baseline concentrations of LPH $(\beta=0.45, p=.002)$ and 4-HNE $(\beta=0.38, p=.005)$ were associated with greater improvement in composite immediate recall among n-3 PUFA-treated patients. No other associations were observed. Conclusions. N-3 PUFA treatment may be more likely to improve immediate recall in patients with greater oxidative stress.

\section{Introduction}

Patients with coronary artery disease (CAD) more commonly demonstrate subtle cognitive deficits $[1,2]$ and are at increased risk for dementia relative to those without CAD $[3,4]$. Decline in immediate recall appears to precede multidomain cognitive decline as both cognitively normal individuals and patients with mild cognitive impairment progress toward dementia [5, 6]. Accordingly, deficits in immediate recall may be important to monitor and remediate in patients with CAD.

As no preventative treatments for dementia currently exist, the potential procognitive effects of omega-3 polyunsaturated fatty acid (n-3 PUFA) supplementation have been investigated by a multitude of clinical trials $[7,8]$, including a recent trial in CAD patients [9]. Results of those trials 
indicate that although n-3 PUFA treatment is generally inefficacious for improving cognition, immediate recall may be a treatment-responsive domain [7]. However, immediate recall response to $\mathrm{n}-3$ PUFA treatment is heterogeneous and this heterogeneity is likely unrelated to study-level differences [7]. These findings suggest that pathophysiological differences between patients may be a potential factor in treatmentresponse variability.

Oxidative stress is a central component of CAD pathophysiology [10] and has been associated with both cognitive deficits and decline [11-13]. N-3 PUFA has demonstrated antioxidant effects in clinical samples [14, 15], and those effects have been related to improvements in memory in animal studies [16]. We hypothesized that n-3 PUFA treatment efficacy for immediate recall might be greater among patients with greater oxidative stress prior to treatment.

This study investigated whether baseline concentrations of the oxidative stress markers lipid hydroperoxides ( $L P H)$, 4-hydroxy-2-nonenal (4-HNE), 8-isoprostane (8-ISO), and malondialdehyde $(M D A)$ were associated with improvement in immediate recall among n-3 PUFA-treated CAD patients.

\section{Methods}

This was a secondary analysis of the cognitive outcomes from the CAD Randomized Omega-3 Trial in Depression (CAROTID), a 12-week, parallel-arm trial of $1.9 \mathrm{~g} /$ day $\mathrm{n}-3$ PUFA treatment (including $1.2 \mathrm{~g} /$ day eicosapentaenoic acid (EPA) and $0.6 \mathrm{~g} /$ day docosahexaenoic acid (DHA)) in CAD patients (NCT00981383) [9]. This study was approved by the Research Ethics Boards of Sunnybrook Health Sciences Centre, University Health Network, and Trillium Health Partners and was conducted according to the principles expressed in the Declaration of Helsinki.

2.1. Patients. Trial inclusion and exclusion criteria are detailed elsewhere [9]. Briefly, patients enrolled in CAROTID were those with evidence of stable CAD (history of myocardial infarction, coronary artery bypass graft, percutaneous transluminal coronary angioplasty, or at least a 50\% stenosis in one or more major coronary artery), aged 45-80 years, male or female, and with the ability to speak and understand English. All eligible patients, with or without depression, were included. Excluded patients were those with a significant acute medical illness, clinically significant cognitive impairment (Standardized Mini-Mental State Examination score $<24$ or a diagnosis of dementia), a neurological condition, unstable angina, or a contraindication to n-3 PUFA supplement use. Antidepressant use was permitted if used at a stable dose for at least 3 months prior to the trial.

2.2. Design. Eligible patients were invited to a prerandomization baseline visit, at which, demographic, anthropomorphic, medical, and medication information was documented. Immediate recall performance was assessed using the immediate recall components of the California Verbal Learning Test, Second Edition [17], and the Brief Visuospatial Memory Test-Revised [18]. Raw scores from each test were adjusted for population norms and the resulting $Z$-scores were used in the analysis. Composite immediate recall was the primary outcome measure and was calculated by the mean of verbal and visuospatial recall $Z$-scores for each patient. Depressive symptom severity was measured using the 17-Item Hamilton Depression Rating Scale (HAM-D) [19] and was accounted for as a covariate. Fasting (12 hours overnight) blood was drawn and processed for analysis of serum lipid peroxidation markers and plasma n-3 PUFAs, which were a planned covariate. Patients were then randomized $(1: 1)$ to receive either $1.9 \mathrm{~g} /$ day $\mathrm{n}-3$ PUFA supplements or placebo for 12 weeks. Composite immediate recall performance was reassessed after 12 weeks.

2.3. Analysis of Lipid Peroxidation Markers and n-3 PUFA. All samples for each lipid peroxidation marker were analysed in the same batch to eliminate batch-to-batch variability. LPH was measured using a colorimetric LPH assay kit with slight modifications (Cayman Chemical, item number 705002). LPH radicals were extracted from serum into chloroform and then mixed with the LPH assay kit. This kit measured the LPH radicals directly through redox reactions with ferrous ions, which were detected using thiocyanate as the chromogen. Each sample was plated in triplicate and the average serum $\mathrm{LPH}$ concentration for each sample was determined by converting the resulting absorbance $(500 \mathrm{~nm})$ of each sample in spectrophotometry to $\mu \mathrm{mol} / \mathrm{L}$ using a hydroperoxide concentration standard. The sensitivity of our technique is between 0.5 and $45.00 \mu \mathrm{mol} / \mathrm{L}$ of hydroperoxides. The interassay coefficient of variation was between $0.0 \%$ and $17.2 \%$.

4-HNE protein adducts, via Michael addition to lysine, histidine, or cysteine, were measured using ELISA (STA838; Cell Biolabs). 4-HNE in serum was mixed with bovine serum albumin, incubated overnight, and then mixed with anti-HNE polyclonal antibody and a horseradish peroxidase conjugated secondary antibody. Each sample was plated in triplicate and the average 4-HNE concentration for each sample was determined by converting the resulting absorbance of the horseradish peroxidase reaction with a substrate solution in spectrophotometry $(450 \mathrm{~nm})$ to $\mathrm{fmol} / \mu \mathrm{g}$ using a protein standard. The sensitivity of this assay is between 3.9 and $250 \mathrm{fmol} / \mu \mathrm{g}$ of 4 -HNE-bovine serum albumin. The interassay coefficient of variation was between $0.0 \%$ and $25.9 \%$, with $95.3 \%(81 / 85)$ of samples yielding an interassay coefficient of variation lower than $25.0 \%$.

8-ISO was measured using a standard competitive sandwich ELISA (\#516351; Cayman Chemical) according to manufacturer's instructions. Serum 8-ISO was conjugated to acetylcholinesterase, with the complex then bound to rabbit IgG mouse monoclonal antibody in the well of the ELISA plate. Each sample was plated in duplicate and the average 8-ISO concentration for each sample was determined by converting the resulting absorbance of acetylcholinesterase reaction with a substrate solution in spectrophotometry $(412 \mathrm{~nm})$ to $\mathrm{pg} / \mathrm{mL}$ using a protein standard. The sensitivity of this assay is between 0.8 and $500 \mathrm{pg} / \mathrm{mL}$. The interassay coefficient of variation was between $0.0 \%$ and $28.7 \%$, with $98.8 \%(84 / 85)$ of samples yielding an interassay coefficient of variation lower than $25.0 \%$. 
Serum MDA concentrations (Cayman; item number 700870) were measured based on the absorbance of thiobarbituric acid reactive substances in spectrophotometry $(540 \mathrm{~nm})$. MDA-thiobarbituric acid adducts were generated in acidic and high temperature $\left(90-100^{\circ} \mathrm{C}\right)$ conditions. Each sample was plated in duplicate and the average MDA concentration for each sample was determined by converting the resulting absorbance to $\mu \mathrm{mol} / \mathrm{L}$ using a thiobarbituric acid standard. The sensitivity of this assay is between 0 and $50 \mu \mathrm{mol} / \mathrm{L}$. The interassay coefficient of variation was between $0.0 \%$ and $22.6 \%$.

Plasma concentrations of EPA, DHA, and the n-6 PUFA, arachidonic acid $(A A)$, were measured by gas chromatography as previously described [20]. All analyses were performed blinded to treatment allocation and patient characteristics.

2.4. Statistical Analyses. Data missing due to dropout were imputed using the multiple imputation method [21], and the resulting dataset was used for the primary analysis. Baseline concentrations of LPH, 4-HNE, 8-ISO, and MDA were each assessed as predictors of change in composite immediate recall $Z$-scores over 12 weeks in both the n-3 PUFA and placebo groups using linear regression. Each model consisted of change in the composite immediate recall $Z$-score as the dependent variable, with baseline composite immediate recall $Z$-score and the lipid peroxidation marker being studied as independent variables. Results for each marker in each treatment group were adjusted for the false discovery rate [22], and only those remaining significant were investigated further.

The ratio of baseline EPA and DHA concentrations to AA concentrations $(E P A+D H A / A A)$ was calculated and included as a planned covariate given the relationships of those fatty acids with cognitive performance [23,24]. Baseline HAM-D score was an additional planned covariate as depressive symptoms may also influence cognitive performance [9].

In post hoc analyses, observed relationships between baseline lipid peroxidation marker concentrations and changes in composite immediate recall $Z$-scores were assessed in the per-protocol subgroup of patients. Additionally, composite immediate recall $Z$-scores were deconstructed into verbal and visuospatial recall $Z$-scores, and relationships with lipid peroxidation markers were explored in each domain.

Statistical models were computed using SPSS statistical software, version 13.0, Chicago, IL, USA, and all analyses were two-tailed.

\section{Results}

As detailed elsewhere [9], 92 patients with CAD were enrolled in CAROTID. Despite adequate treatment compliance, patients using n-3 PUFA did not demonstrate improvement in immediate recall over 12 weeks relative to those using placebo. Of those randomized, 85 patients provided baseline serum lipid peroxidation samples and were included in this study.

Patients in each treatment group were similar (Table 1) with respect to age, sex, years of education, cardiovascular history, medication use, baseline immediate recall performance, baseline EPA + DHA/AA ratio, and baseline concentrations of the lipid peroxidation markers. Mean depressive symptom severity was mild and was similar between groups.

Greater baseline concentrations of LPH and 4-HNE were significantly associated with greater improvement in composite immediate recall among patients receiving 12 weeks of n-3 PUFA treatment (Table 2, Figure 1), after correcting for multiple comparisons. Baseline concentrations of 8-ISO and MDA were not associated with changes in composite immediate recall in n-3 PUFA-treated patients. Similarly, there were no associations between baseline LPH, 4-HNE, or 8-ISO and changes in composite immediate recall among patients using placebo. A significant relationship between greater baseline MDA concentrations and decline in composite immediate recall over 12 weeks was observed in the placebo group; however, it did not survive correction for the false discovery rate.

Greater baseline concentrations of LPH (B (SE) $=0.02$ (0.01), $\beta=0.39, p=.013$ ), and 4-HNE (B (SE) $=0.01$ (0.01), $\beta=0.29, p=.047$ ) remained associated with greater improvement in composite immediate recall over 12 weeks of n-3 PUFA treatment after adjusting for baseline EPA + DHA/AA ratios and baseline HAM-D scores as covariates.

3.1. Post Hoc Analyses. In the per-protocol subgroup of patients treated with n-3 PUFAs $(n=34)$, greater baseline concentrations of LPH (B (SE) $=0.03(0.01), \beta=0.47, p=$ $.002)$ and 4 -HNE (B (SE) $=0.01(0.01), \beta=0.39, p=.013)$ remained significantly associated with greater improvement in composite immediate recall with 12 weeks of n-3 PUFA treatment.

Deconstructing composite immediate recall into verbal recall and visuospatial recall revealed that greater baseline concentrations of LPH (verbal recall: $\mathrm{B}(\mathrm{SE})=0.01(0.01), \beta=$ $0.18, p=.31$; visuospatial recall: $\mathrm{B}(\mathrm{SE})=0.04(0.01), \beta=0.46$, $p=.001)$ and 4-HNE (verbal recall: $\mathrm{B}(\mathrm{SE})=0.01(0.01), \beta=$ $0.20, p=.17$; visuospatial recall: $\mathrm{B}(\mathrm{SE})=0.02(0.01), \beta=0.34$, $p=.014)$ were particularly associated with improvement in visuospatial recall after n-3 PUFA treatment.

\section{Discussion}

This secondary analysis of cognitive outcomes from the CAROTID trial found that higher baseline concentrations of the lipid peroxidation markers LPH and 4-HNE, indicating greater pretreatment levels of oxidative stress, were associated with greater improvements in immediate recall in CAD patients after 12 weeks of n-3 PUFA treatment. The fact that no such associations were observed among patients using placebo and that known predictors of cognitive change, such as age, years of education, and cardiovascular risk factors [25], were balanced at baseline between the treatment groups accords with a potential relationship between oxidative stress and n-3 PUFA treatment efficacy.

To our knowledge, this is the first study to examine the relationship between baseline markers of oxidative stress and n-3 PUFA efficacy on memory or other cognitive domains. However, markers of oxidative stress and inflammation 
TABLE 1: Baseline participant characteristics.

\begin{tabular}{|c|c|c|c|c|}
\hline Variable, mean (SE) & Placebo $(n=46)$ & N-3 PUFA $(n=39)$ & $F / \chi^{2}$ & $p$ value \\
\hline Age & $59.8(1.2)$ & $62.6(1.4)$ & 2.13 & .15 \\
\hline Male, $\%$ & 74 & 80 & 0.44 & .51 \\
\hline Education, yrs & $15.7(0.5)$ & $14.9(0.6)$ & 1.17 & .28 \\
\hline \multicolumn{5}{|l|}{ Cardiovascular history } \\
\hline Event/procedure* & & & 0.80 & .85 \\
\hline MI, \% & 39 & 31 & & \\
\hline PTCA, \% & 37 & 38 & & \\
\hline $\mathrm{CABG}, \%$ & 22 & 28 & & \\
\hline Vascular risk factors, \# of $5^{* *}$ & $2.8(0.2)$ & $3.1(0.2)$ & 0.89 & .35 \\
\hline $\mathrm{VO}_{2}$ peak, $\%$ age and sex norm & $73 \%(24 \%)$ & $73 \%(20 \%)$ & 0.03 & .86 \\
\hline \multicolumn{5}{|l|}{ Medications } \\
\hline Antidiabetic, \% & 13 & 26 & 2.17 & .14 \\
\hline Antihypertensive, $\%$ & 69 & 82 & 1.86 & .17 \\
\hline Anti-inflammatory, \% & 4 & 2 & 0.22 & .64 \\
\hline Platelet inhibitor, \% & 98 & 92 & 1.43 & .21 \\
\hline Statin, \% & 100 & 98 & 1.16 & .28 \\
\hline \multicolumn{5}{|l|}{ Psychometric performance } \\
\hline Composite recall, $Z$-score & $0.23(0.16)$ & $0.06(0.16)$ & 0.61 & .44 \\
\hline Verbal recall, $Z$-score & $0.37(0.16)$ & $0.30(0.16)$ & 0.10 & .75 \\
\hline Visuospatial recall, Z-score & $0.08(0.19)$ & $-0.19(0.18)$ & 1.02 & .32 \\
\hline HAM-D score & $7.6(0.8)$ & $6.8(1.1)$ & 0.32 & .57 \\
\hline \multicolumn{5}{|l|}{ Blood markers } \\
\hline Plasma EPA + DHA/AA ratio & $0.34(0.03)$ & $0.33(0.02)$ & 0.12 & .73 \\
\hline Serum LPH, $\mu \mathrm{mol} / \mathrm{L}$ & $18.2(1.9)$ & $17.7(2.0)$ & 0.03 & .87 \\
\hline Serum $4-\mathrm{HNE}, \mathrm{fmol} / \mu \mathrm{g}$ & $46.4(2.2)$ & $44.5(2.7)$ & 0.27 & .60 \\
\hline Serum 8-ISO, pg/mL & $0.13(0.01)$ & $0.11(0.01)$ & 1.06 & .31 \\
\hline Serum MDA, $\mu \mathrm{mol} / \mathrm{L}$ & $0.04(<0.01)$ & $0.04(<0.01)$ & 0.02 & .89 \\
\hline
\end{tabular}

SE, standard error; N-3 PUFA, $\mathrm{n}-3$ polyunsaturated fatty acid; $F, F$-statistic of analysis of variance; $\chi^{2}$, chi-squared test statistic; MI, myocardial infarction; PTCA, percutaneous transluminal coronary angioplasty; CABG, coronary artery bypass graft; $\mathrm{VO}_{2}$ peak, peak volume of oxygen uptake during cardiac stress test; HAM-D, Hamilton Depression Rating Scale; EPA, eicosapentaenoic acid; DHA, docosahexaenoic acid; AA, arachidonic acid; LPH, lipid hydroperoxides; 4-HNE, 4-hydroxy-2-nonenal; 8-ISO, 8-isoprostane; MDA, malondialdehyde. * Patients may have had both an event and one or more procedures. ** Vascular risk factors: hypertension, obesity (body mass index $\geq 30$ ), dyslipidemia, diabetes mellitus, and smoking.

TABLE 2: Associations between baseline lipid peroxidation marker concentrations and changes in composite immediate recall $Z$-scores over 12 weeks by treatment group.

\begin{tabular}{|c|c|c|c|c|c|c|}
\hline \multirow{2}{*}{ Outcome } & \multicolumn{3}{|c|}{ Placebo $(n=46)$} & \multicolumn{3}{|c|}{ n-3 PUFA $(n=39)$} \\
\hline & $\mathrm{B}(\mathrm{SE})$ & $\beta$ & $p$ value & $\mathrm{B}(\mathrm{SE})$ & $\beta$ & $p$ value \\
\hline$\overline{\mathrm{LPH}}$ & $0.01(0.01)$ & 0.05 & .70 & $0.25(0.01)$ & 0.45 & $.002^{*}$ \\
\hline 4-HNE & $-0.01(0.01)$ & -0.07 & .59 & $0.14(0.01)$ & 0.38 & $.005^{*}$ \\
\hline 8-ISO & $-1.07(1.26)$ & -0.12 & .39 & $-0.65(2.00)$ & -0.05 & .75 \\
\hline MDA & $-12.40(5.18)$ & -0.32 & .017 & $-4.28(4.70)$ & -0.14 & .36 \\
\hline
\end{tabular}

Note. B and $\beta$ are the unstandardized and standardized regression coefficients, respectively. SE is the standard error of the B coefficient. * Indicates that the result remained statistically significant after adjustment for false discovery rate (first threshold at $<.0125$, second threshold at <.025; Benjamini \& Hochberg). Significant $p$ values are shown to three decimal places for clarity.

have been previously associated with n-3 PUFA treatment response in other conditions, such as depression [26, 27], which aligns with our hypothesis that oxidative stress may be relevant to the success of n-3 PUFA supplementation and may underlie its variable efficacy in previous trials [7-9].

Mechanistically, n-3 PUFAs appear to have antioxidant effects [28]. In particular, EPA and DHA have been shown to reduce the production of hydroxyl and superoxide radicals, in turn reducing the production of downstream reactive oxygen species that can attack lipids and proteins. Such antioxidant effects have been observed in both animal studies [29-32] and clinical studies involving patients with mild cognitive deficits [15]. Furthermore, animal studies have shown that n3 PUFA antioxidant effects correlate with improved memory 

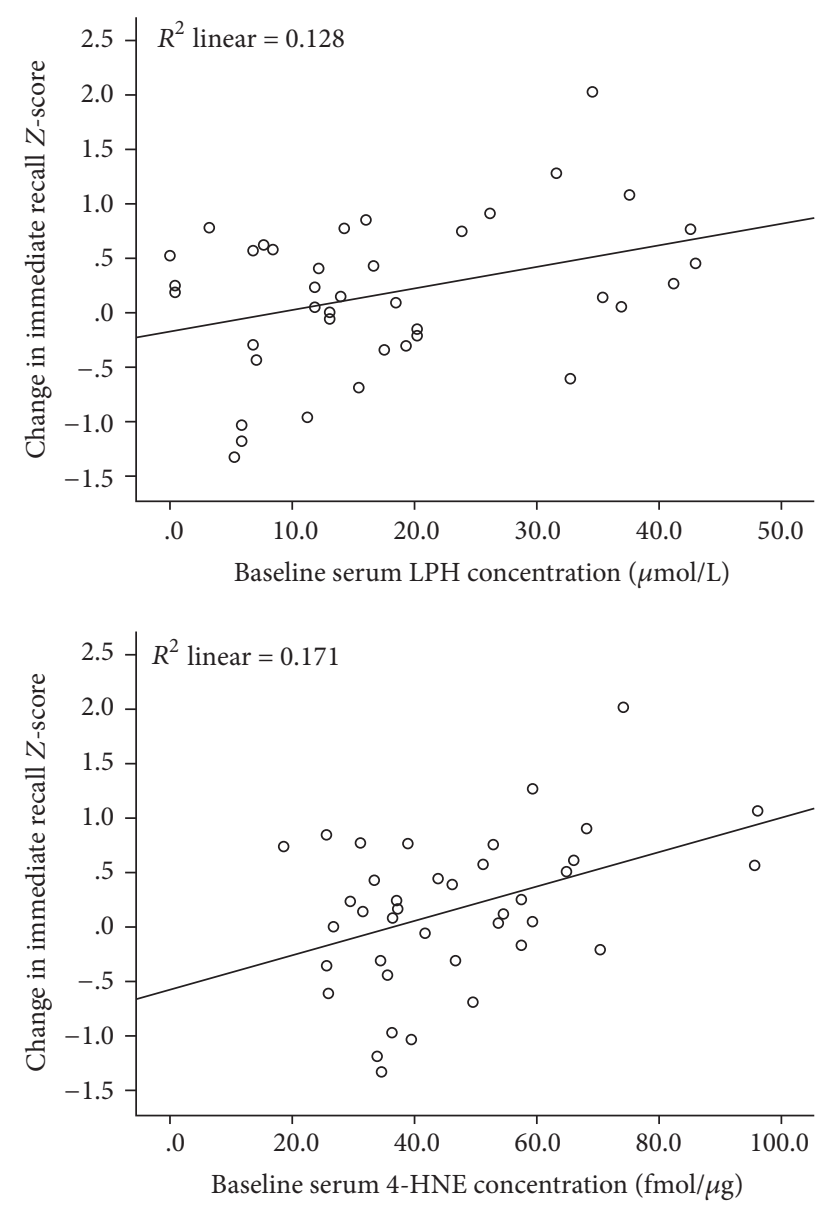

FIGURE 1: Associations of baseline LPH and 4-HNE concentrations with changes in composite immediate recall $Z$-scores over 12 weeks of n-3 PUFA treatment $(n=39)$.

performance $[16,33,34]$. It is unclear why LPH and 4-HNE were the only markers associated with n-3 PUFA treatment benefits. Preclinical studies have not clearly revealed the conditions under which LPH may differentially convert to 4HNE, MDA, or 8-ISO in vivo and so we cannot speculate on the reason for our findings in this clinical sample. However, all four markers have been linked with memory deficits [35-38], as well as n-3 PUFA antioxidant effects [29-32] in animal studies, suggesting the potential for their involvement. Replication of our study may clarify which markers are most relevant to n-3 PUFA treatment effects on memory.

4.1. Strengths and Limitations. A strength of this study is that important predictors of cognitive change such as age, years of education, and cardiovascular risk factors were balanced between the treatment groups and were therefore not required as covariates. As such, we could examine the relationship between baseline oxidative stress and cognitive change in each treatment group using a relatively small sample size. However, the small size precluded examination of that relationship in a more powerful interaction (oxidative stress $\times$ treatment $\times$ time) model combining both treatment groups. Another strength was our ability to account for depressive symptoms, which have been previously shown to influence cognitive performance $[9,39]$ and are highly prevalent in the CAD population [40].

A limitation of this study was that the included patients, despite demonstrating a range of baseline immediate recall performance, were all cognitively healthy and may therefore have been limited in their potential for cognitive change. However, the measures of immediate recall used in this study both provide a high ceiling for potential scores and none of the included patients approached the maximum at either study visit. Moreover, such a limitation would apply equally to both treatment groups and is unlikely to have confounded our findings. How baseline oxidative stress may be associated with cognitive changes among patients with greater cognitive deficits remains to be investigated. Finally, our findings are limited to the domain of immediate recall and to the CAD population. As such, they are not necessarily generalizable to cognitively healthy individuals without cardiovascular disease or patients with cognitive deficits secondary to other vascular diseases or nonvascular causes.

\section{Conclusions}

After adjusting for multiple comparisons and potential confounders, greater baseline concentrations of LPH and 4-HNE were associated with greater improvement in immediate recall performance among CAD patients after 12 weeks of n3 PUFA treatment. Our finding may clarify the variability in cognitive response to n-3 PUFA observed in previous trials. Future research is warranted to assess whether these markers have clinical utility.

\section{Conflicts of Interest}

The authors declare that there are no conflicts of interest regarding the publication of this article.

\section{Acknowledgments}

The authors acknowledge operating funds from the Ontario Mental Health Foundation and the Canadian Institutes of Health Research (CIHR) MOP 114913 to Krista L. Lanctôt and Nathan Herrmann. Gustavo Scola received postdoctoral support from the CAMH Foundation. Graham Mazereeuw received a CIHR Training Program in Neurodegenerative Lipidomics graduate scholarship as well as an Ontario Graduate Scholarship.

\section{References}

[1] A. Salzwedel, M.-D. Heidler, K. Haubold et al., "Prevalence of mild cognitive impairment in employable patients after acute coronary event in cardiac rehabilitation," Vascular Health and Risk Management, vol. 13, pp. 55-60, 2017.

[2] J. Saxton, G. Ratcliff, A. Newman et al., "Cognitive test performance and presence of subclinical cardiovascular disease in the cardiovascular health study," Neuroepidemiology, vol. 19, no. 6, pp. 312-319, 2000. 
[3] L. Zheng, W. J. MacK, H. C. Chui et al., "Coronary artery disease is associated with cognitive decline independent of changes on magnetic resonance imaging in cognitively normal elderly adults," Journal of the American Geriatrics Society, vol. 60, no. 3, pp. 499-504, 2012.

[4] E. Kuźma, J. Airdrie, T. J. Littlejohns et al., "Coronary Artery Bypass Graft Surgery and Dementia Risk in the Cardiovascular Health Study," Alzheimer Disease \& Associated Disorders, vol. 31, no. 2, pp. 120-127, 2017.

[5] M. Bilgel, Y. An, A. Lang et al., "Trajectories of Alzheimer disease-related cognitive measures in a longitudinal sample," Alzheimer's \& Dementia, vol. 10, no. 6, pp. 735-742, 2014.

[6] S. Cloutier, H. Chertkow, M.-J. Kergoat, S. Gauthier, and S. Belleville, "Patterns of cognitive decline prior to dementia in persons with mild cognitive impairment," Journal of Alzheimer's Disease, vol. 47, no. 4, pp. 901-913, 2015.

[7] G. Mazereeuw, K. L. Lanctôt, S. A. Chau, W. Swardfager, and N. Herrmann, "Effects of omega-3 fatty acids on cognitive performance: A meta-analysis," Neurobiology of Aging, vol. 33, no. 7, pp. 1482-e29, 2012.

[8] K. Yurko-Mauro, D. D. Alexander, and M. E. Van Elswyk, "Docosahexaenoic acid and adult memory: A systematic review and meta-analysis," PLoS ONE, vol. 10, no. 3, Article ID e0120391, 2015.

[9] G. Mazereeuw, N. Herrmann, P. I. Oh et al., "Omega-3 fatty acids, depressive symptoms, and cognitive performance in patients with coronary artery disease," Journal of Clinical Psychopharmacology, vol. 36, no. 5, pp. 436-444, 2016.

[10] C. B. Nemeroff and P. J. Goldschmidt-Clermont, "Heartache and heartbreak-the link between depression and cardiovascular disease," Nature Reviews Cardiology, vol. 9, no. 9, pp. 526-539, 2012.

[11] M. Schrag, C. Mueller, M. Zabel et al., "Oxidative stress in blood in Alzheimer's disease and mild cognitive impairment: a metaanalysis," Neurobiology of Disease, vol. 59, pp. 100-110, 2013.

[12] W. R. Markesbery, R. J. Kryscio, M. A. Lovell, and J. D. Morrow, "Lipid peroxidation is an early event in the brain in amnestic mild cognitive impairment," Annals of Neurology, vol. 58, no. 5, pp. 730-735, 2005.

[13] S. L. Harrison, A. J. M. de Craen, N. Kerse et al., "Predicting Risk of Cognitive Decline in Very Old Adults Using Three Models: The Framingham Stroke Risk Profile; the Cardiovascular Risk Factors, Aging, and Dementia Model; and Oxi-Inflammatory Biomarkers," Journal of the American Geriatrics Society, vol. 65, no. 2, pp. 381-389, 2017.

[14] F. Azizi-Soleiman, S. Jazayeri, S. Eghtesadi et al., "Effects of pure eicosapentaenoic and docosahexaenoic acids on oxidative stress, inflammation and body fat mass in patients with type 2 diabetes," International Journal of Preventive Medicine, vol. 4, no. 8, pp. 922-928, 2013.

[15] L. K. Lee, S. Shahar, N. Rajab, N. A. M. Yusoff, R. A. Jamal, and S. M. Then, "The role of long chain omega-3 polyunsaturated fatty acids in reducing lipid peroxidation among elderly patients with mild cognitive impairment: A case-control study," The Journal of Nutritional Biochemistry, vol. 24, no. 5, pp. 803-808, 2013.

[16] H.-M. Su, "Mechanisms of n-3 fatty acid-mediated development and maintenance of learning memory performance," The Journal of Nutritional Biochemistry, vol. 21, no. 5, pp. 364-373, 2010.

[17] V. Hachinski, C. Iadecola, R. C. Petersen et al., "National Institute of Neurological Disorders and Stroke-Canadian Stroke Network vascular cognitive impairment harmonization standards," Stroke, vol. 37, no. 9, pp. 2220-2241, 2006.
[18] R. Benedict, Brief Visuospatial Memory Test-Revised: Professional Manual, Psychological Assessment Resources, Inc, Lutz, FL, USA, 1997.

[19] J. B. Williams, "A structured interview guide for the hamilton depression rating scale," Archives of General Psychiatry, vol. 45, no. 8, pp. 742-747, 1988.

[20] S. A. Abdelmagid, S. E. Clarke, D. E. Nielsen et al., "Comprehensive profiling of plasma fatty acid concentrations in young healthy canadian adults," PLoS ONE, vol. 10, no. 2, Article ID e0116195, 2015.

[21] D. B. Rubin, Multiple Imputation for Nonresponse in Surveys, John Wiley \& Sons, New York, NY, USA, 1987.

[22] Y. Benjamini and Y. Hochberg, "Controlling the false discovery rate: a practical and powerful approach to multiple testing," Journal of the Royal Statistical Society B: Methodological, vol. 57, no. 1, pp. 289-300, 1995.

[23] S. Yagi, T. Hara, R. Ueno et al., "Serum concentration of eicosapentaenoic acid is associated with cognitive function in patients with coronary artery disease," Nutrition Journal, vol. 13, no. 1, article no. 112, 2015.

[24] J. Nishihira, T. Tokashiki, Y. Higashiuesato et al., "Associations between Serum Omega-3 Fatty Acid Levels and Cognitive Functions among Community-Dwelling Octogenarians in Okinawa, Japan: The KOCOA Study," Journal of Alzheimer's Disease, vol. 51, no. 3, pp. 857-866, 2016.

[25] M. Baumgart, H. M. Snyder, M. C. Carrillo, S. Fazio, H. Kim, and H. Johns, "Summary of the evidence on modifiable risk factors for cognitive decline and dementia: a population-based perspective," Alzheimer's \& Dementia, vol. 11, no. 6, pp. 718-726, 2015.

[26] M. H. Rapaport, A. A. Nierenberg, P. J. Schettler et al., "Inflammation as a predictive biomarker for response to omega3 fatty acids in major depressive disorder: A proof-of-concept study," Molecular Psychiatry, vol. 21, no. 1, pp. 71-79, 2016.

[27] G. Mazereeuw, N. Herrmann, A. C. Andreazza et al., "Oxidative stress predicts depressive symptom changes with omega-3 fatty acid treatment in coronary artery disease patients," Brain, Behavior, and Immunity, vol. 60, pp. 136-141, 2017.

[28] E. Giordano and F. Visioli, "Long-chain omega 3 fatty acids: molecular bases of potential antioxidant actions," Prostaglandins, Leukotrienes and Essential Fatty Acids, vol. 90, no. 1, pp. $1-4,2014$.

[29] M. Sarsilmaz, A. Songur, H. Özyurt et al., "Potential role of dietary $\omega-3$ essential fatty acids on some oxidant/antioxidant parameters in rats' corpus striatum," Prostaglandins, Leukotrienes and Essential Fatty Acids, vol. 69, no. 4, pp. 253-259, 2003.

[30] K. Casós, M. C. Zaragozá, N. Zarkovic et al., "A fish oil-rich diet reduces vascular oxidative stress in apo $\mathrm{E}^{-1-}$ mice," Free Radical Research, vol. 44, no. 7, pp. 821-829, 2010.

[31] V. Brahmbhatt, M. Oliveira, M. Briand et al., "Protective effects of dietary EPA and DHA on ischemia-reperfusion-induced intestinal stress," The Journal of Nutritional Biochemistry, vol. 24, no. 1, pp. 104-111, 2013.

[32] G. Abdukeyum, A. Owen, T. Larkin, and P. McLennan, "UpRegulation of Mitochondrial Antioxidant Superoxide Dismutase Underpins Persistent Cardiac Nutritional-Preconditioning by Long Chain n-3 Polyunsaturated Fatty Acids in the Rat," Journal of Clinical Medicine, vol. 5, no. 3, p. 32, 2016.

[33] S. Yadav, K. V. Mitha, M. T. Shenoy, S. Mayannavar, and B. Ganaraja, "Beneficial effect of Omega-3 polyunsaturated fatty acids on neurosensorial impairments and oxidative status 
in Streptozotocin induced diabetic rats," Indian Journal of Physiology and Pharmacology, vol. 58, no. 4, pp. 346-353, 2014.

[34] N. Okabe, T. Nakamura, T. Toyoshima, O. Miyamoto, F. Lu, and T. Itano, "Eicosapentaenoic acid prevents memory impairment after ischemia by inhibiting inflammatory response and oxidative damage," Journal of Stroke and Cerebrovascular Diseases, vol. 20, no. 3, pp. 188-195, 2011.

[35] I. Ohsawa, K. Nishimaki, Y. Murakami, Y. Suzuki, M. Ishikawa, and S. Ohta, "Age-dependent neurodegeneration accompanying memory loss in transgenic mice defective in mitochondrial aldehyde dehydrogenase 2 activity," The Journal of Neuroscience, vol. 28, no. 24, pp. 6239-6249, 2008.

[36] R. Yang, Q. Wang, L. Min, R. Sui, J. Li, and X. Liu, "Monosialoanglioside improves memory deficits and relieves oxidative stress in the hippocampus of rat model of Alzheimer's disease," Neurological Sciences, vol. 34, no. 8, pp. 1447-1451, 2013.

[37] H. Sato, T. Takahashi, K. Sumitani, H. Takatsu, and S. Urano, "Glucocorticoid generates ROS to induce oxidative injury in the hippocampus, leading to impairment of cognitive function of rats," Journal of Clinical Biochemistry and Nutrition, vol. 47, no. 3, pp. 224-232, 2010.

[38] X. H. Cai, Y. H. Zhou, C. X. Zhang, L. G. Hu, X. F. Fan, C. C. Li et al., "Chronic intermittent hypoxia exposure induces memory impairment in growing rats," Acta Neurobiologiae Experimentalis, vol. 70, no. 3, pp. 279-287, 2010.

[39] L. M. McDermott and K. P. Ebmeier, "A meta-analysis of depression severity and cognitive function," Journal of Affective Disorders, vol. 119, no. 1-3, pp. 1-8, 2009.

[40] B. Rudisch and C. B. Nemeroff, "Epidemiology of comorbid coronary artery disease and depression," Biological Psychiatry, vol. 54, no. 3, pp. 227-240, 2003. 


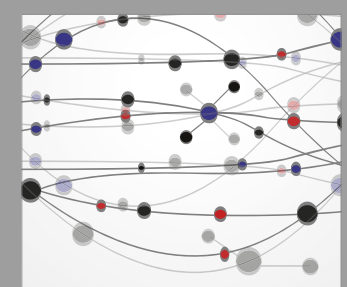

The Scientific World Journal
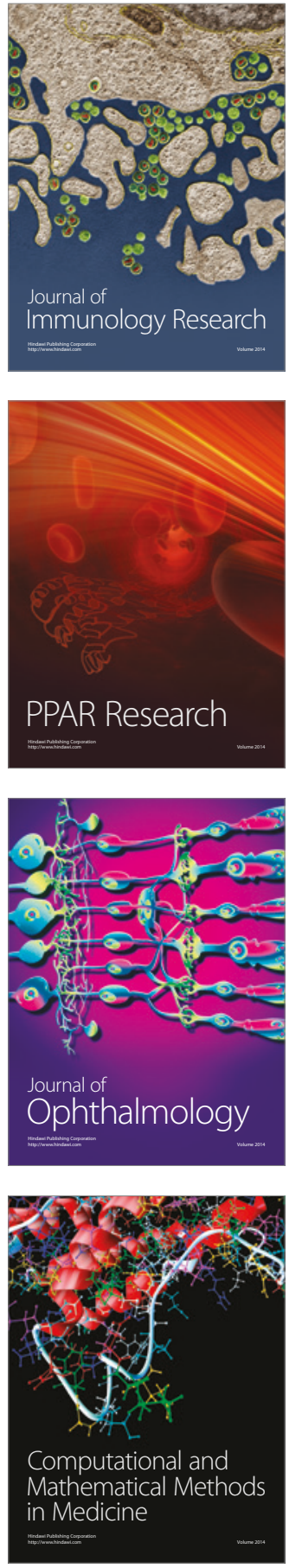

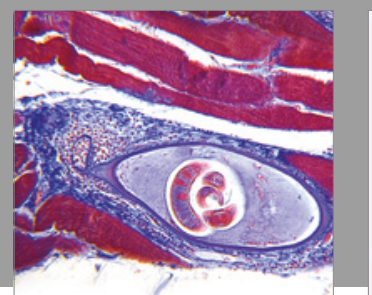

Gastroenterology Research and Practice
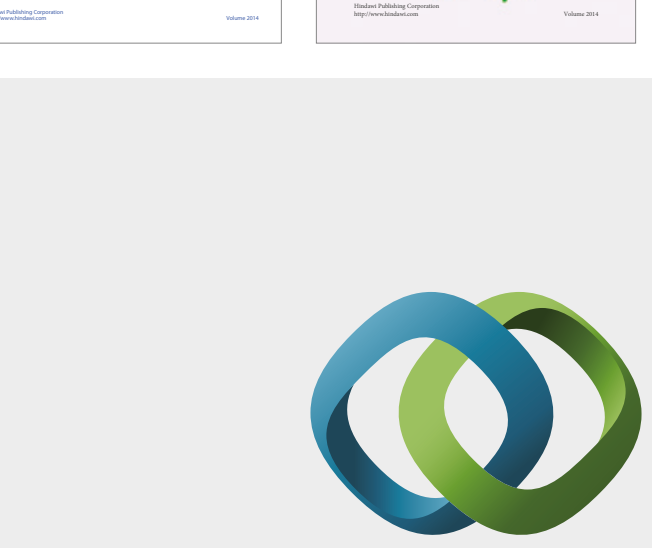

\section{Hindawi}

Submit your manuscripts at

https://www.hindawi.com
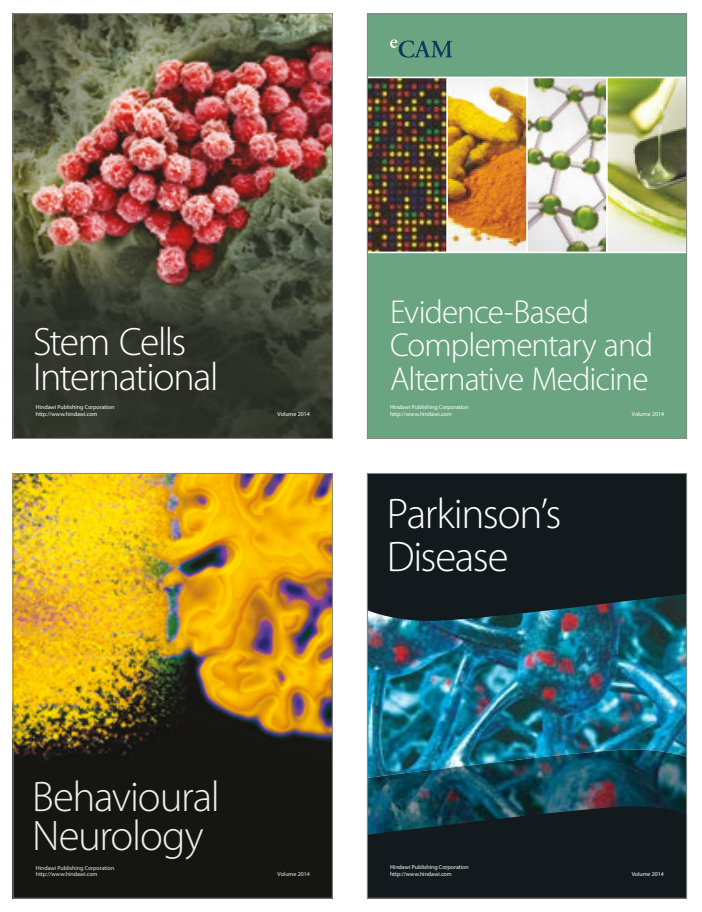
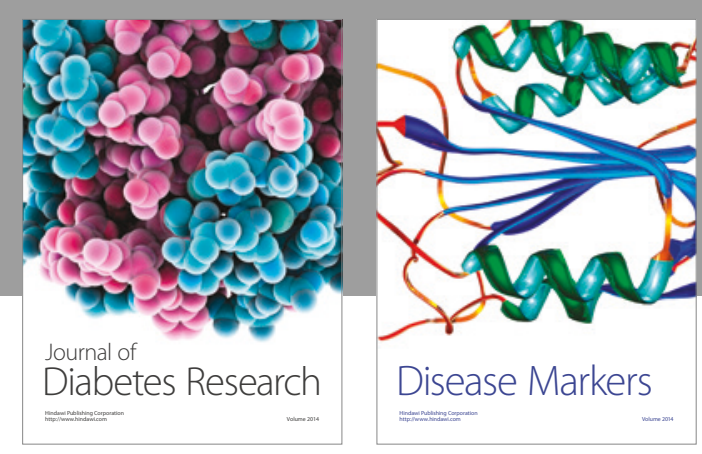

Disease Markers
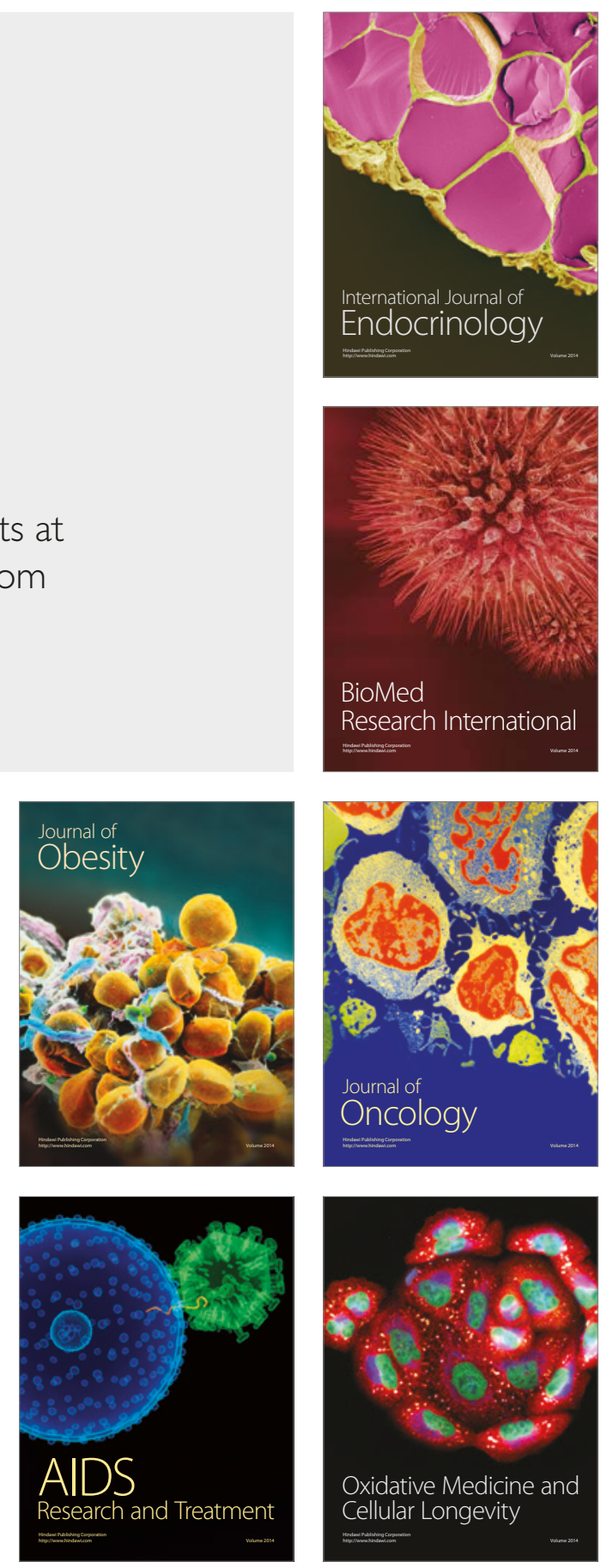